\title{
Retractorless surgery for a pineal region tumor through an occipital transtentorial approach
}

\author{
Naoyuki Nakao, MD, PhD \\ Department of Neurological Surgery, Wakayama Medical University, Wakayama, Japan
}

This video demonstrates surgical techniques of the occipital transtentorial approach to a pineal region tumor without using a fixed brain retractor, which may cause functional impairment or even tissue injury to the occipital visual cortex. There are several ways to facilitate retractorless surgery through this approach. A lateral-semiprone positioning of the patient can induce gravity retraction. The brain is relaxed by draining CSF fluid through lumbar drainage or lateral ventricular tap in the case of obstructive hydrocephalus. Dynamic retraction with handheld instruments after extensive dissection of the deep venous system, including basal veins, can provide a sufficient working space.

The video can be found here: https://youtu.be/kQvEHiNcRow.

KEYWORDS pineal tumor; basal vein of Rosenthal; great vein of Galen; retractorless surgery; occipital; transtentorial approach; video 